\title{
ACTIVIDAD POLINIZADORA Y PREFERENCIA FLORAL DE Bombus spp. (HYMENOPTERA: APIDAE) PRESENTES EN UNA CERCA VIVA
}

\section{POLLINATION ACTIVITY AND FLORAL PREFERENCES OF Bombus spp. (HYMENOPTERA: APIDAE) PRESENT IN A HEDGE}

\author{
Lorena Téllez-Farfán ${ }^{1}$; Francisco J. Posada-Flórez ${ }^{2}$
}

\begin{abstract}
${ }^{1}$ Estudiante de Ciencias Ambientales. Universidad de Ciencias Aplicadas y Ambientales U.D.C.A, Calle 222 No. 55-37, Bogotá, D.C., Colombia. tllezlorena@gmail.com; ${ }^{2}$ Ing. Agrónomo, Entomólogo Ph.D. Facultad de Ingeniería Agronómica, Universidad de Ciencias Aplicadas y Ambientales U.D.C.A, hasta junio de 2012, fjavierposada@hotmail.com
\end{abstract}

Rev. U.D.C.A Act. \& Div. Cient. 16(2): 359-367, Julio-Diciembre, 2013

\section{RESUMEN}

Las especies de Bombus son agentes polinizadores de plantas en la agricultura y de la flora silvestre y contribuyen con este servicio a la producción de alimentos y al mantenimiento de la biodiversidad. Por esto es importante realizar investigaciones para conocerlos, conservarlos y aprovecharlos, en programas de polinización. Este estudio, se realizó en predios de la U.D.C.A, localizada al norte de la Sabana de Bogotá, Colombia y tuvo como objetivo identificar las especies de Bombus que visitan el campus universitario, registrar los horarios en que presentan mayor actividad de visita a las flores y reportar cómo esta actividad es afectada por los cambios de la temperatura ambiental. Adicionalmente, se determinó la flora y se registró el número de visitas realizadas a las flores. Se detectaron dos especies de Bombus: B. robustus y $B$. atratus, siendo $B$. atratus la más abundante en el campus universitario. Los registros mostraron dos picos de actividad a temperaturas, que oscilaron entre 16 y $19^{\circ} \mathrm{C}$. En relación con la flora visitada, se detectaron 18 especies visitadas por ambas especies de Bombus, siendo el trébol rojo, Trifolium pratense (Fabaceae), la especie que presentó el mayor número de visitas.

Palabras clave: Actividad en campo, Bombus atratus, condiciones ambientales, especies vegetales, polinización.

\section{SUMMARY}

Bombus species are pollinators of agricultural crops and wild flora, contributing to food production and the maintenance of biodiversity. Thus, it is important to perform research aimed to get a better knowledge to preserve them and use them in crop pollination programs. This study was performed at U.D.C.A's campus, located in the northern part of the Sabana of Bogota, Colombia. The objectives of this study were to identify the Bombus species found visiting flowers at the University campus, to register the hours of major activity, and to report how their activity was affected by daily environmental temperature changes. Additionally, the flora visited was identified and the number of visits to the flora was recorded. Two Bombus species were found: $B$. robustus and $B$. atratus, the latest being the most abundant at the University campus. Two peaks of activity at temperatures that oscillated between $16^{\circ} \mathrm{C}$ and $19^{\circ} \mathrm{C}$ were observed. Eighteen plant species were visited being clover, Trifolium pretense (Fabaceae) the most visited one.

Key words: Bombus atratus, forage activity, host plants, pollination, environmental condition.

\section{INTRODUCCIÓN}

La polinización es el proceso más importante en la vida de las plantas con reproducción sexual, ya que permite la mezcla de los genes que repercutirán en la herencia de la nueva planta, favoreciendo así su eficacia biológica. Algunas especies son capaces de auto polinizarse, pero otras requieren polen de otra planta para poder reproducirse, por lo que necesitan de un agente que lo transporte hasta el órgano receptor femenino de la planta. Diversos organismos y algunos agentes abióticos realizan la polinización, pero los que más se destacan, por realizar esta función, son los insectos (Delaplane \& Mayer, 2000; James \& Pitts-Singer, 2008; Abrol, 
2012).Entre ellos, los polinizadores por excelencia son los himenópteros, específicamente las abejas, debido a su estricta dieta de néctar y polen para el mantenimiento de la colonia (Delaplane \& Mayer, 2000; James \& Pitts-Singer, 2008). Se ha calculado que el $75 \%$ de los cultivos y el $80 \%$ de todas las plantas con flores dependen de la polinización por factores bióticos o animales (Randall \& Radloff, 2011).

Las abejas contribuyen ampliamente a la economía de los seres humanos, gracias a la polinización que realizan, ya que a partir de esta se optimizan diversos productos vegetales, como los frutos y las semillas (Delaplane \& Mayer, 2000; Aldana et al. 2007). El hecho que las abejas participen en la formación de productos vegetales implica que favorece la reproducción vegetal y, por lo tanto, cooperan a mantener la diversidad de especies vegetales.

A pesar de la escaza información e investigación en abejas de Colombia, se han podido identificar 564 especies, pertenecientes a seis familias, 13 subfamilias, 23 tribus y 55 géneros (Nates-Parra \& González, 2000). Los abejorros del género Bombus pertenecen a la familia Apidae, subfamilia Apinae y tribu Bombini. Éstos, se han convertido en los mayores polinizadores en cultivos de tomate y en invernaderos de zonas templadas, debido a que presentan la capacidad de "vibrar" las flores para facilitar el desprendimiento del polen, tienen un hábito de vida social, lo que implica mayor cantidad potencial de individuos forrajeros y la pilosidad en su cuerpo, característica propia de las abejas de la familia Apidae, que contribuye al transporte del polen. Adicionalmente, dependiendo de la especie, presentan glosa más larga que la abeja común, Apis mellifera, por lo que utilizan un grupo más amplio de flora (Prŷs-Jones \& Corbet, 1991; Delaplane \& Mayer, 2000).

Liévano et al. (1994) estudiaron el género Bombus, único representante de la tribu Bombini en Colombia e identificaron nueve especies, tratando temas como taxonomía (Liévano et al. 1994) y algo de su biología (Nates-Parra \& González, 2000); otros autores, como Abrahamovich \& Díaz (2002), registraron doce especies, basándose en los registros de otros autores, como Moure \& Sakagami (1962), Milliron (1973) y Abrahamovich \& Díaz (2001), entre otros. En algunas zonas aledañas a Bogotá (Colombia), se encontraron cinco especies de Bombus, que tienen distribución estrictamente andina; de estas, solamente $B$. atratus tiene distribución altitudinal amplia (Nates-Parra et al. 2006).

El tiempo de actividad diaria, épocas climáticas y las preferencias alimentarias de Bombus en Colombia, se han estudiado poco, aunque existen algunos trabajos que proporcionan un acercamiento. Por ejemplo, las especies de Bombus identificadas por Nates-Parra et al. (2006), se alimentan de vegetación exótica, obteniendo néctar y polen, lo que ten- dría una repercusión seria en la competencia entre especies vegetales foráneas y nativas (Morales, 2007). Abrahamovich et al. (2007) realizaron un registro de las familias vegetales asociadas a las especies de Bombus en Argentina, encontrándose una amplia diversidad de familias de plantas.

En la Universidad de Ciencias Aplicadas y Ambientales U.D.C.A, en Bogotá, Colombia, se han observado individuos de Bombus pecoreando en varias especies vegetales, como: el trébol rojo (Trifolium pratense L, Fabaceae), el trébol blanco (T. repens L., Fabaceae), el diente de león (Taraxacum officinale Weber, Compositae) y el nabo forrajero (Raphanus sativus L., Brassicaceae) (Posada-Flórez, 2011). En Colombia, aún se requiere realizar estudios en donde se identifique la preferencia de Bombus por las especies vegetales que visita, información que sería útil para la conservación del género y de las especies vegetales que polinizan.

Adicionalmente, se han realizado estudios sobre la eficacia de la polinización por parte de Bombus en frutales, como tomate, ají y lulo y su influencia en la producción de alimentos de calidad, obteniendo resultados que demuestran que la propiedad de los alimentos aumenta cuando han sido polinizados por Bombus (Kwon \& Saeed, 2003; Almanza, 2007; Aldana et al. 2007). Lo anterior confirma la importancia del polinizador Bombus, en la seguridad alimentaria de la humanidad.

En cuanto al conocimiento de la actividad de Bombus en regiones tropicales, existen pocos estudios sobre su actividad en campo, en donde se determine, por especie, los horarios de mayor actividad con relación a la temperatura, debido a su condición de ectotermos, dado que se les puede observar en zonas frías, como en los ecosistemas de páramo (SDA, 2012). Morales-Rozo et al. (2006) determinaron que la mayor actividad forrajera de $B$. atratus en una zona de La Valvanera (Chía, Cundinamarca), se encuentra entre las 10:00 y las 14.00 horas, con una temperatura ambiental promedio de $22^{\circ} \mathrm{C}$, resultados que requieren ser confirmados con mayor número de muestreos, debido a las pocas repeticiones de observación directa de la actividad forrajera.

Adicionalmente, Kwon \& Saeed (2003) realizaron un estudio del efecto de la temperatura en la actividad polinizadora de $B$. terrestris bajo condiciones de invernadero, pero la información que aporta su estudio al comportamiento de las especies colombianas es mínimo, ya que esta especie no es nativa del país (Williams, 2008) y a que las condiciones de invernadero son diferentes a las de los espacios al aire libre, que son el hábitat natural de Bombus.

Dada su importancia como polinizadores y a que existen vacíos de información es necesario conservar la fauna de Bombus y para lograrlo es imprescindible conocerlos. En la U.D.C.A y en la zona de influencia del humedal Torca-Guay 
maral, aún no se tiene registro de las especies de Bombus que visitan su flora, por lo que este trabajo tuvo como objetivo reconocer las especies de Bombus que visitan la flora del campus norte de la Universidad, determinar la flora que utilizan y relacionar su actividad con la temperatura ambiente. Se espera que este estudio pueda servir de base para posteriores investigaciones, que contribuyan al conocimiento de Bombus spp. en Colombia, información que se puede utilizar para su conservación y aprovechamiento de su función como polinizadores eficientes.

\section{MATERIALES Y MÉTODOS}

La U.D.C.A, se encuentra localizada al norte de Bogotá (Colombia), entre las coordenadas $4^{\circ} 47^{\prime} 57^{\prime \prime} N-74^{\circ} 02^{\prime} 40^{\prime \prime} W$. Sus condiciones ecológicas son $2.570 \mathrm{msnm}$, precipitación media anual de $860 \mathrm{~mm}$ con régimen bimodal y temperatura media anual de $14,5^{\circ} \mathrm{C}$ (Salazar et al. 2010), factores que caracterizan la zona agroecológica, como bosque seco montano bajo (bs-MB), propio de los altiplanos andinos (Espinal \& Montenegro, 1977). El campus norte de la U.D.C.A hace parte del territorio de tipo rural de Bogotá y es aledaña al humedal de Torca-Guaymaral, zona recientemente declarada Reserva Forestal (CAR, 2011).

En la U.D.C.A existe vegetación de diversas especies y estratos, entre nativas y exóticas. En el campus norte, a lo largo del perímetro donde se han sembrado especies arbóreas y arbustivas nativas, también se presenta vegetación herbácea exótica. Las especies exóticas de mayor abundancia son el diente de león, Taraxacum officinale (Compositae), trébol blanco, Trifolium repens, trébol rojo, T. pratense (Fabaceae) y nabo forrajero, Raphanus sativus (Cruciferae). También, se encuentran otras especies vegetales, como raque, Vallea stipularis (Elaeocarpaceae), astromelia, Alstroemelia aurantiaca (Alstroemeliaceae), mora silvestre, Rubus bogotensis (Rosaceae) y calabaza, Cucurbita sp. (Cucurbitaceae), entre otras (observaciones de los autores).

La evaluación de la actividad de Bombus en el campus norte de la U.D.C.A, se realizó en dos épocas climáticas: lluviosa (marzo) y seca (junio y julio). En ambos períodos, se ejecutaron durante un día por semana, cuatro evaluaciones diarias, en días sin lluvia, ya que estas alteran la actividad de Bombus. Los muestreos, se practicaron entre las 6:00 y las 18:00 horas, durante 20 minutos cada uno y consistieron en el recorrido de la zona escogida, la cual, tuvo $400 \mathrm{~m}$ de longitud por $20 \mathrm{~m}$ de ancho aproximadamente $\left(8000 \mathrm{~m}^{2}\right)$. En cada recorrido, se tomaron los datos de hora, especie de Bombus pecoreando, su abundancia y la flora visitada. Para el conteo de la abundancia, los especímenes no se marcaron ni capturaron, pudiéndose haber realizado el conteo sobre el mismo individuo, en diferente tiempo.
La determinación taxonómica de los individuos de Bombus, se realizó basándose en los patrones de coloración; en el caso de no haber certeza sobre la especie, se colectaba el individuo para la confirmación y se liberaba. En tres casos, los individuos ingresaron a la colección entomológica de la U.D.C.A, para confirmación con patrones de coloración y de genitalias. Adicionalmente, se tomaron fotografías de individuos de Bombus en la flora visitada, durante los recorridos de los muestreos.

Los datos de temperatura ambiental por hora, se tomaron de la página de la Red de Calidad de Aire de la Secretaría Distrital de Ambiente de Bogotá, estación Guaymaral, que se ubica en la Escuela Colombiana de Ingeniería (447'1.0”N$74^{\circ} 2^{\prime} 37^{\prime}$ 'W), a una altura de dos metros sobre la superficie terrestre y a una distancia aproximada de $2,3 \mathrm{~km}$ del campus norte de la U.D.C.A.

Otros datos climáticos, como radiación solar y viento, no se analizaron, ya que no fueron parte del objetivo del estudio. Igualmente, la precipitación no se analizó, porque no se presentó lluvia en los días en que se realizaron los conteos y se previó, para garantizar que ocurriera la actividad de Bombus.

Los resultados, se analizaron calculando las estadísticas descriptivas y se realizaron pruebas de análisis de varianza entre los conteos de Bombus y los registros de temperatura, entre la época de lluvia y seca. Adicionalmente, se calculó el coeficiente de correlación, para establecer el tipo de relación por hora entre el conteo de la actividad de Bombus y el promedio de la temperatura, para los días de muestreo (SAS Institute Inc., 2006). Además, se determinaron los horarios en que se presentó mayor ocurrencia de Bombus spp. durante el día, de acuerdo con la época climática y sus preferencias por las especies vegetales, mediante el conteo de las visitas a las especies florales.

\section{RESULTADOS Y DISCUSIÓN}

En la evaluación realizada en la época de lluvia, en los cuatro días de muestreo, se registraron 249 conteos de Bombus, los cuales, pertenecían a las especies $B$. atratus $(n=246$; $98,8 \%$ ) y $B$. robustus $(\mathrm{n}=3 ; 1,2 \%)$, mientras que en la época seca, se contaron 256 individuos, todos pertenecientes a $B$. atratus. La actividad de Bombus por día presentó, en ambas evaluaciones, el mismo patrón, lo que al parecer indica que la actividad obedece al efecto de las variables de temperatura, a través del día y a la disponibilidad de vegetación con flores, que ofrecen alimento (Figura 1, 2, Tabla 1).

Se reconocieron dos especies de Bombus en el campus de la U.D.C.A, B. atratus y B. robustus, de las nueve especies registradas para Colombia (Liévano et al. 1994). B. atratus fue la más abundante $(n=502 ; 98,8 \%)$, por lo que los re- 

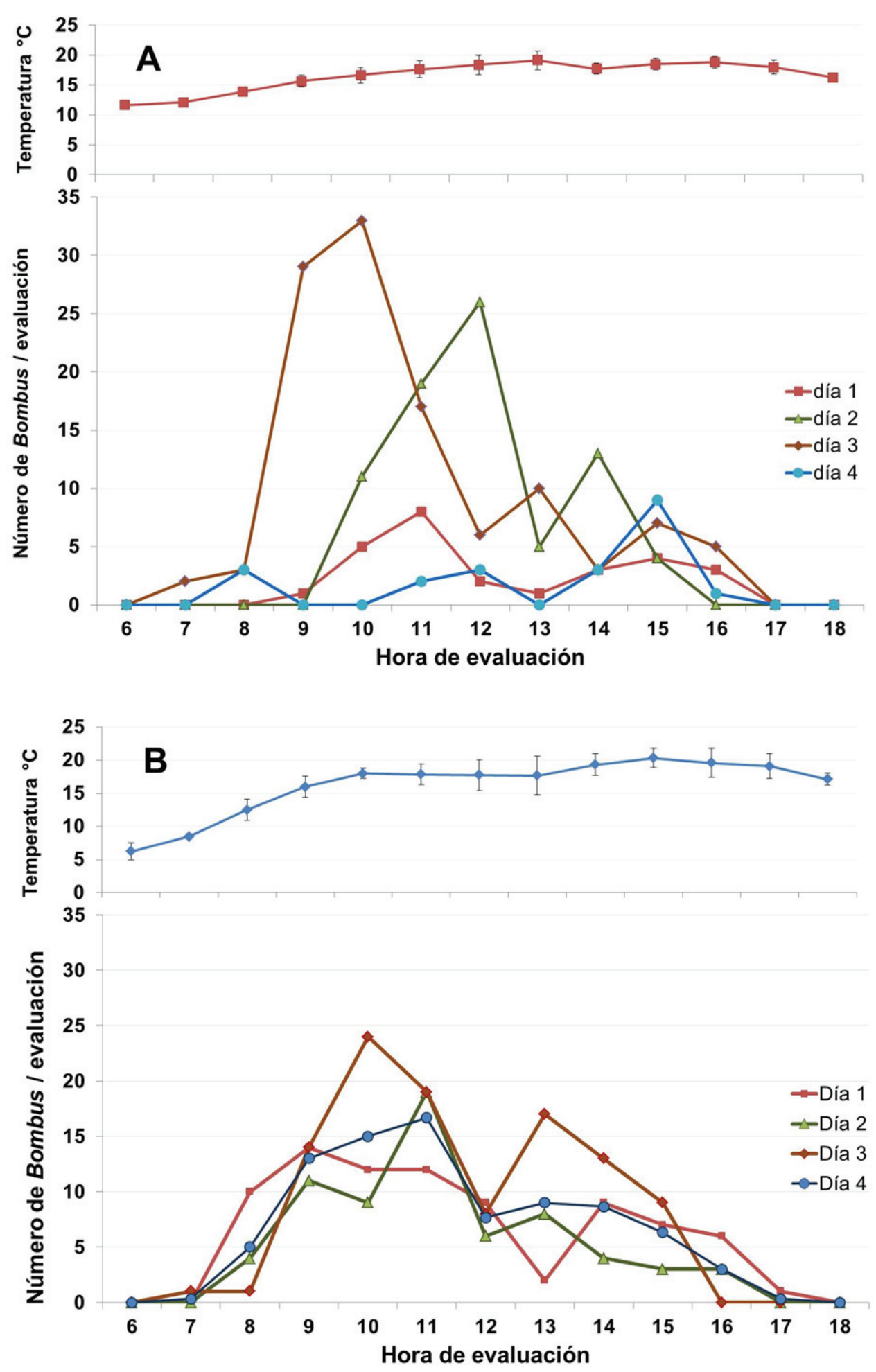

Figura 1. Distribución de los conteos de Bombus en la U.D.C.A, con respecto a la hora del día y el promedio de la temperatura, para los días de evaluación. A) Evaluacion en época de lluvia y B) Evaluación época seca. 

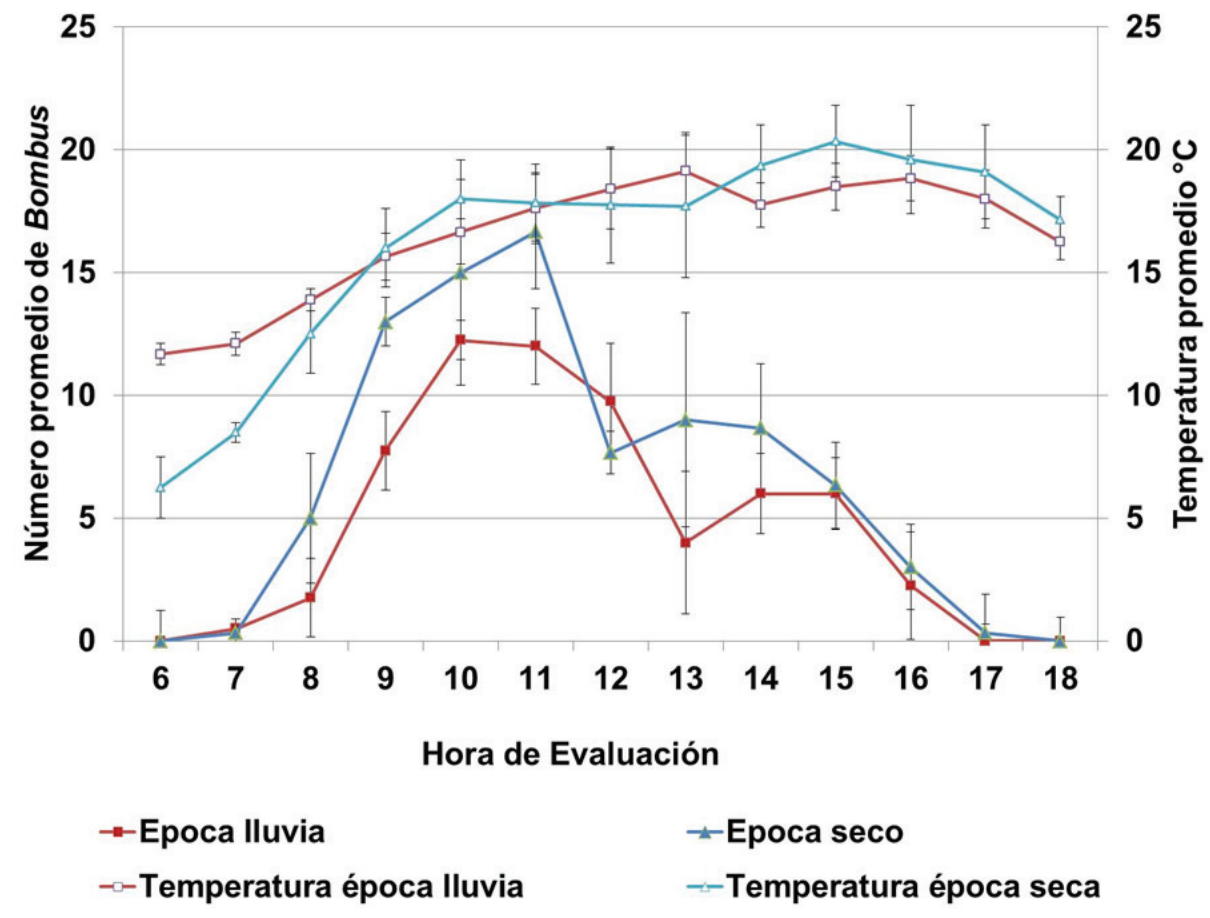

Figura 2. Actividad promedio del pecoreo de Bombus spp., durante dos evaluaciones en época de lluvia y seca, en predios de la U.D.C.A, en contraste con la temperatura promedio para los días de muestreo.

sultados de actividad fueron liderados por esta especie que, de acuerdo con las observaciones de campo (Figuras $1 \mathrm{y}$ 2) y con el estudio realizado por Morales-Rozo et al. (2006), parece ser la especie que domina la zona norte de Bogotá. B. robustus $(\mathrm{n}=3 ; 1,2 \%)$ sólo se observó esporádicamente, su abundancia en el campus fue mínima y únicamente se contaron en las tres primeras evaluaciones en la época de lluvia. Sobre la ecología de esta especie existe escasez de información.

Los resultados del muestreo pueden indicar que su hábitat está amenazado o que están tratando de establecerse, ya que en la zona de muestreo se están realizando prácticas de conservación, como es el establecimiento de parcelas de cercas vivas con flora de especies nativas.

La actividad de Bombus en ambas evaluaciones presentó dos picos, que corresponden a los momentos de incremento de las mayores temperaturas (Figuras 1 y 2 ). La temperatura mínima, a la que $B$. atratus y $B$. robustus comenzaron su actividad de pecoreo, fue de $10,9^{\circ} \mathrm{C}$, entre las $7: 00 \mathrm{~h}$ y $8: 00 \mathrm{~h}$ y la temperatura máxima en la que se observó actividad fue de $23^{\circ} \mathrm{C}$, entre las $13: 00$ y $14: 00 \mathrm{~h}$.

En época de lluvias, $B$. atratus presentó dos picos de abundancia en actividad forrajera, entre las 10:00h y las11:00h y las 14:00h y las 15:00h. El pico de mayor actividad, para esta especie, ocurrió a una temperatura que osciló entre los 16 y $17^{\circ} \mathrm{C}$. Conforme aumentaba la temperatura, la actividad de Bombus decreció; sin embargo, el segundo pico de actividad, se presentó a una temperatura entre los 18 y $19^{\circ} \mathrm{C}$, aunque a la máxima temperatura, mayor a los $19^{\circ} \mathrm{C}$, la actividad disminuyó drásticamente a unos pocos individuos, por tiempo de conteo (Figura 2).

Entre las 14:00h y 15:00h, la temperatura decreció, lo que al parecer, estimuló el leve aumento de la actividad de $B$. atratus. Conforme aumentó de nuevo la temperatura, la actividad de Bombus volvió a decaer (Figura 2).

La baja actividad en campo por parte Bombus en los periodos de altas temperaturas, se debe, probablemente, a su necesidad de mantener las temperaturas constantes dentro del nido y a nivel individual, pues el hecho de volar incrementa la temperatura corporal, lo que no es favorecido por un incremento en la temperatura ambiente (Alford, 1978). De forma similar, al incrementarse la temperatura ambiente, las obreras del nido deben airearlo para mantener una temperatura favorable para el desarrollo de la cría, razón por la cual, disminuye la actividad de pecoreo (O’Donell \& Foster, 2001; Weindenmüler, 2004). 
Tabla 1. Especies florales visitadas por las dos especies de Bombus en la U.D.C.A.

\begin{tabular}{|c|c|c|c|c|}
\hline Nombre común & Nombre científico & Familia & $\begin{array}{l}\text { No. Visitas } \\
\text { observadas }\end{array}$ & $\begin{array}{c}\text { Especie de } \\
\text { Bombus }\end{array}$ \\
\hline Trébol rojo & Trifolium pratense & Fabaceae & 329 & B. atratus \\
\hline Nabo forrajero & Raphanus sativus & Brassicaceae & 49 & $\begin{array}{l}\text { B. atratus, } \\
\text { B. robustus }\end{array}$ \\
\hline Trébol blanco & Trifolium repens & Fabaceae & 37 & B. atratus \\
\hline Papa & Solanum tuberosum & Solanaceae & 19 & B. atratus \\
\hline Retamo liso & Teline monspesulana & Fabaceae & 19 & B. atratus \\
\hline Astromelia & Alstroemeria aurantiaca & Alstroemeliaceae & 18 & B. atratus \\
\hline Brócoli & Brassica oleracea italica & Brassicaceae & 12 & B. atratus \\
\hline Diente de león & Taraxacum officinale & Asteraceae & 8 & B. atratus \\
\hline Mora silvestre & Rubus sp. & Rosaceae & 7 & B. atratus \\
\hline Raque & Vallea stipularis & Elaeocarpaceae & 3 & $\begin{array}{l}\text { B. atratus, } \\
\text { B. robustus }\end{array}$ \\
\hline Calabaza & Cucurbita sp. & Cucurbitaceae & 2 & B. atratus \\
\hline Estatice & Limoniun sinuatum & Plumbaginaceae & 1 & B. atratus \\
\hline Geranio & Geranium pelargonium & Geraniaceae & 1 & B. atratus \\
\hline Curuba & Passiflora mollisima & Passifloraceae & 1 & B. atratus \\
\hline \multirow[t]{2}{*}{ Siete cueros } & Tibouchina lepidota & Melastomataceae & 1 & B. atratus \\
\hline & & Lamiaceae & 1 & B. atratus \\
\hline Duraznillo & Abatia parviflora & Flacourtiaceae & 1 & $\begin{array}{l}\text { B. atratus, } \\
\text { B. robustus }\end{array}$ \\
\hline Lupino & Lupinus polyphyllus & Fabaceae & 1 & B. atratus \\
\hline
\end{tabular}

Es importante resaltar que en el segundo pico de actividad, entre las $14: 00 \mathrm{~h}$ y $16: 00 \mathrm{~h}$, se observaron cuatro reinas pecoreando, todas de $B$. atratus, lo que puede dar algún indicio de la preferencia en los horarios de pecoreo por parte de las reinas; esta información puede contribuir a planear las actividades de investigación sobre el establecimiento natural de nidos de Bombus en campo o para realizar actividades de captura de reinas, con el propósito de iniciar nidos y realizar investigación sobre crías artificiales.

En época seca, el comportamiento de $B$. atratus fue similar al de la época de lluvias; presentó dos picos de actividad, antes del mediodía, lo mostró en el mismo periodo que en época de lluvias, entre las 10:00 y 11:00h, a una temperatura cercana a los $18^{\circ} \mathrm{C}$, pero la actividad decayó una hora antes que la de la otra época, a pesar que la temperatura se mantuvo con poca variación. El segundo pico de actividad, después del mediodía, se inició y decayó también una hora antes, en comparación con la actividad de la época de lluvias. Este segundo pico, se dio entre temperaturas promedio que oscilaron entre los 19,35 y $20,35^{\circ} \mathrm{C}$ (Figura 2).
La hora de inicio y cese de la actividad de las especies de Bombus, encontradas en este estudio, fue variable en todos los conteos y épocas de evaluación. En dos conteos, se registró actividad a las 7:00 horas, mientras que en los otros conteos, se registró a partir de las 8:00 horas.

En relación con el cese de actividad, se registró a las 16:00 horas, para tres conteos, todos en época de lluvias y, a las 17:00 horas, para un conteo, este en época seca; en el resto de las evaluaciones, en esta última época, entre las 17 y 18 horas, no se realizó algún registro. Estos resultados indican que la actividad de Bombus en campo, bajo las condiciones ambientales del norte de la Sabana de Bogotá, se extiende por ocho a diez horas. Esta información es importante tenerla en cuenta para utilizarla en la fenología floral de las especies vegetales, en las que se intente utilizar Bombus para la polinización en condiciones de campo abierto y para la aplicación de medidas sanitarias en los cultivos, con el propósito de evitar la intoxicación de los polinizadores, al usar insecticidas. 
Comparando los conteos en época de lluvia y seca, se encontró mayor actividad en dos conteos en época de lluvia; esto, probablemente se presentó, porque en la época seca se observó mayor actividad de labores agrícolas y de mantenimiento de los prados, donde cortan la vegetación arvense, con lo que se disminuye la oferta de plantas en floración (Hatfield et al. 2012). Estas prácticas agrícolas afectan la actividad de las visitas a las flores por parte de sus polinizadores y, por lo tanto, la abundancia de estos, que, probablemente, vuelan a otros lugares más distantes, en busca de alimento.

En el análisis estadístico, no se encontró diferencia entre las evaluaciones realizadas en época de lluvia y seca $(\mathrm{GL}=1,89$; $\mathrm{F}=1,30 ; \mathrm{P}=0,25)$. Igualmente, no se encontraron diferencias estadísticas entre la temperatura registrada en la época de lluvia y seca. La correlación entre el conteo de Bombus y el registro de la temperatura presentó una correlación baja $(r=0,34)$, lo que indica que la actividad de Bombus es compleja, ya que puede depender de la interacción de las variables abióticas y de la actividad antrópica.

B. robustus inició actividad entre las 7:00h y 8:00h, con temperatura mínima promedio de $12,1^{\circ} \mathrm{C}$. Se observaron individuos entre las 12:00h-13:00h y las 14:00h-15:00h, cuando la temperatura ambiente oscilaba entre los 17,8 y $18,4^{\circ} \mathrm{C}$. Sobre esta especie es necesario realizar más observaciones, para tener un mayor conocimiento de su biología.

En el estudio de Kwon \& Saeed (2003), las altas temperaturas disminuyen la actividad de $B$. terrestris y la actividad de forrajeo comienza desde muy temprano, desde las 7:00h a 8:00h, a una temperatura de $25,8^{\circ} \mathrm{C}$. Conforme aumenta la temperatura, la actividad disminuye. En este caso, a partir de los $19^{\circ} \mathrm{C}$, la actividad de $B$. atratus disminuyó, lo que lleva a inferir que las altas temperaturas, mayores a $19^{\circ} \mathrm{C}$, no favorecen la actividad de $B$. atratus, en la Sabana de Bogotá. Aun así, la respuesta a los cambios de temperatura difiere entre individuos, siendo unos más tolerantes a las altas temperaturas, mientras que otros no. Esto concuerda, con el estudio de O'Donell \& Foster (2001) y Weindenmüler (2004), en donde se comprueba que cada individuo, dentro de una colonia, presenta diferencias en el umbral de respuesta ante un cambio en el ambiente, siendo algunos más tolerantes, con umbrales más altos y otros no tanto, con umbrales más bajos.

En la interpretación de los factores ambientales sobre las poblaciones de Bombus en campo, se debe ser cuidadoso y no generalizar tendencias. Los estudios de Bombus en campo, en relación con actividad de vuelo y visita de flores corrientemente, se realizan en zonas templadas y en épocas de verano, donde el fotoperiodo y la temperatura se comportan diferente al trópico, donde estos factores son menos variables, porque se presentan con una duración aproximada de 12 horas, lo que influye en la actividad de Bombus y en la fenología de las plantas.

En cuanto a la flora visitada por Bombus spp., se encontraron 18 especies vegetales, pertenecientes a 15 familias (Tabla 1). Las especies que más observaciones tuvieron fueron el trébol rojo (T. pratense) $(\mathrm{n}=329,64,4 \%)$, el nabo forrajero $(R$. sativus, ) ( $n=49,9,6 \%)$, el trébol blanco (T. repens,) $(n=37$, $7,2 \%)$, la papa (S. tuberosum) $(n=19,3,7 \%)$, el retamo liso $(T$. monspesulana) $(n=19,3,7 \%)$ y las astromelias (A. aurantiaceae) $(n=18 ; 3,5 \%)$ (Tabla 1$)$. De estos resultados, se deduce que $B$. atratus prefiere especies foráneas en comparación con las nativas, en donde se observaron visitas, lo que podría ser una desventaja, en el caso de promover la propagación de las especies vegetales nativas, debido al bajo número de observaciones en estas. Lo anterior, se debe confirmar bajo condiciones en las que haya similitud en las abundancias de especies florecidas, para determinar con mayor exactitud las preferencias alimentarias de Bombus spp.

Para $B$. robustus, se deben hacer más observaciones de flora utilizada, pues aparte de observarlo utilizando $V$. stipularis, $R$. sativus y Abatia parviflora, también fue observado en Canna sp., por fuera del periodo de muestreo, especie vegetal, en la cual, se han realizado muy pocos avistamientos de visitas por parte de abejas. Esto conlleva a interpretar que entre especies de Bombus hay diferencias en las preferencias alimentarias, lo que, probablemente, se asocie con sus diferencias en las longitudes de glosa, que significa su alcance al néctar, de acuerdo con el tipo de flor (Prŷs-Jones \& Corbet, 1991).

De las especies encontradas, $B$. atratus es la que muestra un mayor rango de distribución (Nates-Parra, 2005; Nates-Parra et al. 2006) y es la que más fácil se encuentra en la Sabana de Bogotá, lo cual, ha permitido realizar algunos estudios sobre su efecto como polinizador en solanáceas, como el lulo y tomate, demostrándose que la polinización, por parte de Bombus, favorece la formación y el desarrollo de frutos de alta calidad (Almanza, 2007; Aldana et al. 2007).

En la relación de las plantas con la actividad de vuelo y visita de flores de Bombus, se observó que es dependiente de la temperatura y de la luz, ya que plantas, como el diente de león (T. officinale), abre sus flores en la mañana cuando el sol esta iluminando el campo y la temperatura se empieza a elevar. Igualmente, las flores se cierran en la tarde y el momento en que ocurre depende de la luz y la temperatura. Los datos de la actividad de Bombus demostraron que son activos entre las 9:00 y las 16:00 horas, lo cual, se ajusta a la fenología del diente de león. Esto se observó en la Sabana de Bogotá y es importante investigar estos aspectos en más detalle, para establecer si se repite en otras regiones geográficas y si marca un patrón de comportamiento para la acti- 
vidad y la alimentación de Bombus e, igualmente, si afectan la antesis de las flores para liberar el polen y producir néctar.

Conflicto de intereses: El manuscrito fue preparado y revisado con la participación de todos los autores, quienes declaramos que no existe ningún conflicto de intereses, que ponga en riesgo la validez de los resultados presentados.

\section{BIBLIOGRAFÍA}

1. ABRAHAMOVICH, A.H.; DÍAZ, N.B; LUCIA, M. 2007. Identificación de las "abejas sociales" del género Bombus (Hymenotpera: Apidae) presentes en la Argentina: clave pictórica, diagnosis, distribución geográfica y asociaciones florales. Rev. Fac. Agr. La Plata. 106(2):165-176.

2. ABRAHAMOVICH, A.H.; DÍAZ, N.B. 2001. Distribución geográfica de las especies del género Bombus Latreille (Hymenoptera, Apidae) en Argentina. Rev. Bras. Entomol. 45(1):23-26.

3. ABRAHAMOVICH, A.; DÍAZ, N. 2002. Bumble bees of the Neotropical Región (Hymenoptera: Apidae). Biota Col. 3(2):199-214.

4. ABROL, D. 2012. Pollination biology. Springer Science Business Media. 792p.

5. ALFORD, D.V. 1978. The life of the bumblebee. London: Davis-Poynter. 80p.

6. ALMANZA, M.T. 2007. Management of Bombus atratus bumblebees to pollinate lulo (Solanum quitoense L.), a native fruit from the Andes of Colombia. Eco. Developm. Series No. 50. 112p.

7. ALDANA, J.; CURE, J.R.; ALAMANZA, M.T.; VECIL, D.; RODRÍGUEZ, D. 2007. Efecto de Bombus atratus (Hymenoptera: Apidae) sobre la productividad de tomate (Lycopersicon esculentum Mill.) bajo invernadero en la Sabana de Bogotá, Colombia. Agr. Col. 25(1):62-72.

8. CORPORACIÓN AUTÓNOMA REGIONAL DE CUNDINAMARCA -CAR-. 2011. Acuerdo 11 de 2011. Disponible desde internet en <http://www.car.gov. co/?idcategoria $=16738>($ con acceso 07/08/2013).

9. DELAPLANE, K.; MAYER D. 2000. Crop pollination by bees. CABI. New York, USA. 334p.

10. ESPINAL T., L.S.; MONTENEGRO M., E. 1977. Zonas de vida o formaciones vegetales de Colombia. Memoria explicativa sobre el mapa ecológico de Colombia. Instituto Geográfico Agustín Codazzi, IGAC. Departamento Agrológico, Bogotá. 238p.

11. HATFIELD, R.; JEPSEN, S.; MADER, E.; BLACK, S.H.; SHEPHARD, M. 2012. Conserving Bumble Bees: Guidelines for Creating and Managing Habitat or America's Declining Pollinators. The Xerces Society for Invertebrate Conservation. 32p.

12. KWON, Y.; SAEED, S. 2003. Effect of temperature on the foraging activity of Bombus terrestris L. (Hymenoptera: Apidae) on greenhouse hot peeper (Capsicum annuum L.). Appl. Entomol. Zool. 38 (3):275-280.

13. JAMES R.; PITTS-SINGER, T. 2008. Bee pollination in agricultural ecosystems. Oxford University Press Inc. New York, USA. 248p.

14. LIÉVANO A.; OSPINA R.; NATES PARRA, G. 1994. Contribución al conocimiento de la taxonomía del género Bombus en Colombia (Hymenoptera: Apidae). Trianea. 5:221-233.

15. MILLIRON, H.E. 1973. A monograph of the Western Hemisphere bumblebees (Hymenoptera: Apidae; Bombinae). II. The genus Megabombus, Subgenus $\mathrm{Me}$ gabombus. Memoirs Ent. Soc. Canada. 89:81-236.

16. MORALES-ROZO, C.; ROJAS-PRIETO, D.; CURE-HAKIM, J. 2006. Actividad forrajera de un nido silvestre de Bombus atratus Franklin (Hymenoptera: Apidae) en la Sabana de Bogotá. En: III Encuentro Colombiano de Abejas Silvestres. 2006. Laboratorio de Investigaciones en Abejas LABUN, Universidad Nacional de Colombia. Santa Marta, Colombia. p.49.

17. MORALES, C. 2007. Introducción de abejorros (Bombus) no nativos: causas, consecuencias ecológicas y perspectivas. Ecol. Austral 17:51-65.

18. MOURE, J.S.; SAKAGAMI, S.F. 1962. As mamangabas sociais do Brasil (Bombus Latreille) (Hymenoptera, Apoidea). Studia Entomol. 5(1-4):65-194.

19. NATES-PARRA, G. 2005. Abejas corbiculadas de Colombia, Hymenoptera: Apidae. Universidad Nacional de Colombia. Bogotá, Colombia. 156p.

20. NATES PARRA, G.; GONZÁLEZ, V. 2000. Abejas silvestres de Colombia: Por qué y cómo conservarlas. Acta Biol. Col. 5(2):5-37.

21. NATES PARRA, G.; PARRA, A.; RODRÍGUEZ, A.; VAQUE- 
RO P.; VÉLEZ, D. 2006. Abejas silvestres (Hymenoptera: Apoidea) en ecosistemas urbanos: Estudio en la ciudad de Bogotá y sus alrededores. Rev. Col. Entomol. 32(1):77- 84.

22. O'DONELL, S.; FOSTER, R. 2001. Thresholds of Response in Nest thermoregulation by Worker Bumble Bees, Bombus bifarius nearcticus (Hymenoptera: Apidae). Ethology. 107:387-399.

23. POSADA-FLÓREZ, F.J. 2011. Manejo de Polinizadores ¿Qué hace falta? Notas y Noticias Entomológicas. 32(4):23.

24. PRŶYS-JONES, O.; CORBET, S. 1991. Bumblebees. The Richmond Publishing Co. Ltd. England. 92p.

25. RANDALL, H.; RADLOFF, S. 2011. Honeybees of Asia. Springer. 600p.

26. SALAZAR, I.C.; MARTÍNEZ-GRANJA, E.; PEÑA, F.; CABEZAS, M.; GÓMEZ, J. 2010. Identificación del agente causante de la marchitez de proteas (Leucadendron sp.). Rev. U.D.C.A Act. \& Div. Cient. 13(2):79-87.
27. SAS institute Inc. 2006. SAS/Procedures guide versión SAS 9.1.3. SAS Institute Inc., Cary, NC. USA.

28. SECRETARÍA DISTRITAL DE MEDIO AMBIENTE -SDA-. 2012. Parque Ecológico Distrital de Montaña Entrenubes. Disponible desde Internet en: <http://www. secretariadeambiente.gov.co/sda/libreria/pdf/ecosistemas/areas_protegidas/en_a16.pdf $>$ (con acceso 13/05/2012).

29 WEINDENMÜLER, A. 2004. The control of nest climate in bumblebee (Bombus terrestris) colonies: interindividual variability and self- reinforcement in fanning response. Behav. Ecol. 15(1):120-128.

30. WILLIAMS, P. 2008. Bombus bumble bees of the world. Natural History Museum. Disponible desde Internet en: http:/www.nhm.ac.uk/research-curation/ research/projects/bombus/index.html (con acceso 05/04/12).

Recibido: Agosto 17 de 2013

Aceptado: Octubre 31 de 2013.

Como citar:

Téllez-Farfán, L.; Posada-Flórez, F.J. 2013. Actividad polinizadora y preferencia floral de Bombus spp. (Hymenoptera: Apidae) presentes en una cerca viva. Rev. U.D.C.A Act. \& Div. Cient. 16(2): 359-367. 